\title{
A Proof of the Existence of Phase Transitions in the Anisotropic Heisenberg Model
}

\author{
DEREK W. ROBINSON \\ Faculté des Sciences, Luminy, Marseille
}

Received June 13, 1969

\begin{abstract}
It is rigorously proved that the anisotropic Heisenberg spin model, in two or more dimensions, exhibits a first order phase transition at low temperatures and for large anisotropy (strong coupling of the third components of the spins).
\end{abstract}

\section{Introduction}

It is now universally believed that the usual formalism of equilibrium statistical mechanics is structurally rich enough to both predict phase transitions and give information concerning their nature. Unfortunately it is difficult to justify this belief by rigorous mathematical arguments and theoretical proofs of the existence of phase transitions are indeed scarce. In classical statistical mechanics proofs exist for spin systems, or lattice gases, under a variety of different conditions but the only quantum mechanical system for which a transition has been established is the non-interacting Bose gas in three or more dimensions. The purpose of this note is to provide an existence proof for an interacting quantum system, the anisotropic Heisenberg model in two or more dimensions.

The method we use is a variant of an argument devised by Peierls [1] to prove the existence of a first order phase transition in the two dimensional Ising model. In the framework of classical spin systems this method has been generalized and extended by several authors [2-4]. The first point of this note is to present this argument in a manner applicable to quantum spin systems; this requires a slight reformulation of the method in terms of operators but no essential change. Secondly this reformulation is applied to the anisotropic Heisenberg model. This part of our calculation involves estimates of the norms of certain products of non-commuting operators and to establish these estimates we consider the Heisenberg model as a perturbed form of the Ising model, i.e. the corresponding classical system. It is this perturbation theoretic calculation that restricts our results to a range of anisotropies for which the third components of the spins are strongly coupled. 


\section{General Formulation}

Let $Z^{v}$ be a $v$-dimensional cubic lattice and associate with each point $x \in Z^{v}$ a spin $\underline{\sigma}_{x}=\left(\sigma_{x}^{(1)}, \sigma_{x}^{(2)}, \sigma_{x}^{(3)}\right)$. The anisotropic Heisenberg Hamiltonian of a system confined to a finite subset $\Lambda \subset Z^{v}$ is given by

$$
\begin{aligned}
H_{\Lambda}= & \frac{1}{8} \sum_{x, y \in \Lambda}\left[J_{12}(x-y)\left\{\sigma_{x}^{(1)} \sigma_{y}^{(1)}+\sigma_{x}^{(2)} \sigma_{y}^{(2)}\right\}+J_{3}(x-y) \sigma_{x}^{(3)} \sigma_{y}^{(3)}\right] \\
& +h \sum_{x \in A} \sigma_{x}^{(3)}
\end{aligned}
$$

Actually for our purposes it is convenient to avoid the above spin formulation and adopt the equivalent lattice gas notation; we introduce annihilation and creation operators $(2 \times 2$ matrices $) a_{x}, a_{x}^{+}$by

$$
a_{x}=\frac{1}{2}\left(\sigma_{x}^{(1)}-i \sigma_{x}^{(2)}\right), \quad a_{x}^{+}=\frac{1}{2}\left(\sigma_{x}^{(1)}+i \sigma_{x}^{(2)}\right),
$$

and then note that in terms of these operators $H_{A}$ is equivalent to the Hamiltonian $K_{A}=T_{A}+V_{A}-u N_{A}$ where

$$
\begin{gathered}
T_{A}=\frac{1}{2} \sum_{x, y \in \Lambda} J_{12}(x-y) a_{x}^{+} a_{y}, \\
V_{A}=\frac{1}{2} \sum_{x, y \in \Lambda} J_{3}(x-y) a_{x}^{+} a_{x} a_{y}^{+} a_{y}, \\
N_{\Lambda}=\sum_{x \in A} a_{x}^{+} a_{x} \text { and } \mu=-2 h+\sum_{x \in A} J_{3}(x) .
\end{gathered}
$$

[Actually $H_{\Lambda}$ and $K_{\Lambda}$ differ by a term proportional to $N(\Lambda)$, the number of points in $\Lambda$, and a surface term $\sum_{\Lambda}$, i.e. a term such that $\left\|\sum_{\Lambda}\right\| /(N(\Lambda)) \rightarrow 0$ as $\Lambda \rightarrow \infty$; both of these terms are irrelevant to the following discussion]. For simplicity we assume that the interaction potentials $J_{12}$ and $J_{3}$ couple nearest neighbours only, i.e. we take

$$
J_{12}(x-y)=0=J_{3}(x-y)
$$

if $x$ and $y$ are not nearest neighbours, and we adopt the notation

$$
J_{12}(x-y)=J_{12}^{i}, \quad J_{3}(x-y)=J_{3}^{i}, \quad i=1,2, \ldots v
$$

if $x$ and $y$ are nearest neighbours in the direction of the $i$-th coordinate axis. We further introduce the notation

$$
\begin{aligned}
J_{3} & =2 \sum_{i=1}^{v} J_{3}^{i}, \\
\left\|J_{3}\right\| & =2 \sum_{i=1}^{v}\left|J_{3}^{i}\right| \text { and }\left\|J_{12}\right\|=2 \sum_{i=1}^{v}\left|J_{12}^{i}\right| .
\end{aligned}
$$


The states of the finite system form a vector space $\mathscr{H}_{A}$ in which one may introduce a normalized basis, labeled by the subsets of $\Lambda$, in the following manner. The vector $|\theta\rangle$ is defined, up to a phase, by the condition

$$
a_{x}|\theta\rangle=0 \text { for all } x \in \Lambda
$$

and $|X\rangle$ is defined by

$$
|X\rangle=\left(\prod_{x \in X} a_{x}^{+}\right)|\theta\rangle \text { for all } X \subset \Lambda .
$$

We now recall the following standard result.

Proposition. The following limit

$$
P(\beta, \mu)=\lim _{\lambda \rightarrow \infty} \frac{1}{\beta N(\Lambda)} \log \operatorname{Tr}_{\mathscr{H}_{\Lambda}}\left(e^{-\beta K_{\Lambda}}\right)
$$

exists and defines the thermodynamic pressure. The function $(\beta, \mu)$ $\rightarrow P(\beta, \mu)$ is convex, continuous, and satisfies the symmetry principle

$$
P\left(\beta, \mu+\frac{1}{2} J_{3}\right)=P\left(\beta,-\mu+\frac{1}{2} J_{3}\right)+\beta \mu .
$$

Further, if the following condition

$$
\liminf _{\Lambda \rightarrow \infty}\left[\frac{1}{\operatorname{Tr}_{\mathscr{H}_{\Lambda}}\left(e^{\left.-\beta K_{\Lambda}\right)}\right.} \frac{1}{N(\Lambda)} \operatorname{Tr}_{\mathscr{H}_{\Lambda}}\left(e^{-\beta K_{\Lambda}} N_{\Lambda}\right)\right]_{\mu=\frac{1}{2} J_{3}} \leqq \varrho_{0}(\beta)<\frac{1}{2}
$$

is satisfied then $d P(\beta, \mu) / d \mu$ is discontinuous at $\mu=J_{3} / 2$, i.e. the system undergoes a first order phase transition.

In spin language the pressure corresponds to the free energy, the symmetry principle states that this quantity is a symmetric functional of the external field $h$, and the condition given in the proposition is one way of stating that the spontaneous magnetization is non-zero.

Let us consider the spin reversal symmetry a little more closely. If for $x \in Z^{v}$ we introduce the operator $R_{x}$ by

$$
R_{x}=a_{x}^{+}+a_{x}
$$

then we find from the commutation rules $a_{x} a_{x}^{+}+a_{x}^{+} a_{x}=1$ etc. that

$$
R_{x}=R_{x}^{+}, R_{x}^{2}=1, R_{x} a_{x} R_{x}=a_{x}^{+} \quad R_{x} a_{x}^{+} R_{x}=a_{x} .
$$

Corresponding to each finite subset $\Lambda \subset Z^{v}$ we can then introduce the symmetry operator $R_{A}$ by

$$
R_{\Lambda}=\prod_{x \in \Lambda} R_{x} ;
$$


the symmetry property of $P(\beta, \mu)$ follows from noting that

$$
\operatorname{Tr}_{\mathscr{H}_{\Lambda}}\left(e^{-\beta K_{\Lambda}}\right)=\operatorname{Tr}_{\mathscr{H}_{\Lambda}}\left(R_{\Lambda} e^{-\beta K_{\Lambda}} R_{\Lambda}\right)
$$

and then using the above transformation rules to calculate $R_{A} K_{A} R_{A}$.

Next let us consider the reformulation of the Peierls technique which is required to estimate the bond $\varrho_{0}(\beta)$. We will not give a detailed description of this technique but rely upon the formulation, and notation, presented in [4]. To each configuration $X \subset A$ one associates a closed polyhedra $\Gamma(X)$ which splits into disconnected polyhedra $\left\{\gamma_{1}, \ldots, \gamma_{n}\right\}$ which are called cycles. The prescription for obtaining these polyhedra is such that knowledge of the set $\left\{\gamma_{1}, \ldots, \gamma_{n}\right\}$ determines the configuration $X$. For a given cycle $\gamma$ we denote by $|\gamma|_{i}, i=1, \ldots, v$, the number of its faces orthogonal to the $i$-th coordinate axis. One finds that for fixed values of these parameters there are at most

$$
N(\Lambda) \prod_{i=1}^{v} 3^{|y|} i^{-1}
$$

cycles. Further if $n(\gamma)$ denotes the number of lattice points inside of $\gamma$ then with $\Gamma(X)=\left\{\gamma_{1}, \ldots, \gamma_{n}\right\}$ we have

and

$$
N(X) \leqq \sum_{j=1}^{n} n\left(\gamma_{j}\right)
$$

$$
n(\gamma) \leqq \prod_{i=1}^{v}\left(\frac{1}{2}|\gamma|_{i}\right)^{\frac{1}{\nu-1}}
$$

Now let the cycle $\gamma$ belong to $\Gamma(X)$ and let $X^{\prime}$ be such that $\Gamma\left(X^{\prime}\right)$ $=\Gamma(X)-\{\gamma\}$. Further let $I_{\gamma} \subset \Lambda$ denote the set of lattice points inside $\gamma$, let $S_{\gamma} \subset \Lambda$ denote the set of points $x$ which are such that $x \notin I_{\gamma}$ but there is a nearest neighbour $y$ of $x$ such that $y \in I_{\gamma}$, and let $S_{\gamma}^{\prime} \subset x$ denotes the set of points $x \in I_{\gamma}$ such that $x$ has a nearest neighbour $y \notin I_{\gamma}$.

We introduce, with a slight abuse of notation, the symmetry operator

$$
R_{\gamma}=\prod_{x \in I_{\gamma}} R_{x}
$$

and the projection operator

$$
P_{\gamma}=\prod_{x \in S_{\gamma} \cup S_{\gamma}^{\prime}}\left(1-a_{x}^{+} a_{x}\right) .
$$

It is immediately checked from the definition of the cycles $\gamma$ that

$$
R_{\gamma} P_{\gamma}\left|X^{\prime}\right\rangle=|X\rangle \text {. }
$$


Now we have

$$
\begin{aligned}
& \frac{1}{N(\Lambda)} \operatorname{Tr}\left(e^{-\beta K_{\Lambda}} N_{\Lambda}\right)=\sum_{X \subset \Lambda} \frac{N(X)}{N(\Lambda)}\left\langle X\left|e^{-\beta K_{\Lambda}}\right| X\right\rangle \\
& \leqq \sum_{X \subset \Lambda} \sum_{j=1}^{n} \frac{n\left(\gamma_{j}\right)}{N(\Lambda)}\left\langle X\left|e^{-\beta K_{\Lambda}}\right| X\right\rangle \\
& =\sum_{\gamma} \frac{n(\gamma)}{N(\Lambda)} \sum_{X ; \Gamma(X) \ni \gamma}\left\langle X\left|e^{-\beta K_{\Lambda}}\right| X\right\rangle \\
& =\sum_{\gamma} \frac{n(\gamma)}{N(\Lambda)} \sum_{X ; \Gamma(X) \ni \gamma}\left\langle X^{\prime}\left|P_{\gamma} R_{\gamma} e^{-\beta K_{\Lambda}} R_{\gamma} P_{\gamma}\right| X^{\prime}\right\rangle \\
& \leqq \sum_{\gamma} \frac{n(\gamma)}{N(\Lambda)}\left\|e^{\frac{\beta K_{\Lambda}}{2}} P_{\gamma} R_{\gamma} e^{-\beta K_{\Lambda}} R_{\gamma} P_{\gamma} e^{\frac{\beta K_{\Lambda}}{2}}\right\| \sum_{X ; \Gamma(X) \ni \gamma}\left\langle X^{\prime}\left|e^{-\beta K_{\Lambda}}\right| X^{\prime}\right\rangle \\
& \leqq \sum_{\gamma} \frac{n(\gamma)}{N(\Lambda)}\left\|e^{\frac{\beta K_{\Lambda}}{2}} P_{\gamma} R_{\gamma} e^{\frac{-\beta K_{\Lambda}}{2}}\right\|^{2} \operatorname{Tr}_{\mathscr{H}_{\Lambda}}\left(e^{-\beta K_{\Lambda}}\right)
\end{aligned}
$$

Thus

$$
\begin{aligned}
& \frac{1}{\operatorname{Tr}\left(e^{-\beta K_{\Lambda}}\right)} \frac{1}{N(\Lambda)} \operatorname{Tr}\left(e^{-\beta K_{\Lambda}} N_{\Lambda}\right) \\
& \quad \leqq \sum_{|\gamma|_{1} \cdots|\gamma|_{\nu}} \prod_{i=1}^{\nu}\left\{3^{|\gamma|_{i}-1}\left(\frac{1}{2}|\gamma|_{i}\right)^{\frac{1}{\nu-1}}\right\}\left\|e^{\frac{\beta K_{\Lambda}}{2}} P_{\gamma} R_{\gamma} e^{-\frac{\beta K_{\Lambda}}{2}}\right\|^{2}
\end{aligned}
$$

and to establish the existence of a phase transition it remains to obtain a suitable bound on the norm occuring in the last expression at the symmetry point $\mu=\frac{1}{2} J_{3}$.

Before considering the full problem of the Heisenberg model, let us briefly discuss the Ising case $J_{12}=0$. One has then

$$
\left.R_{\gamma} K_{\gamma} R_{\gamma}\right|_{\mu=\frac{1}{2} J_{3}}=\left.K_{\Lambda}\right|_{\mu=\frac{1}{2} J_{3}}-V_{\gamma}-C_{\gamma}
$$

where $V_{\gamma}$ and $C_{\gamma}$ are straightforwardly calculated to be given by

Hence

$$
\begin{aligned}
& V_{\gamma}=\sum_{\substack{x \in \Lambda / I_{\gamma} \\
y \in I_{\gamma}}} J_{3}(x-y)\left(2 a_{x}^{+} a_{x} a_{y}^{+} a_{y}-a_{x}^{+} a_{x}-a_{y}^{+} a_{y}\right), \\
& C_{\gamma}=\frac{1}{2} \sum_{\substack{x \in \Lambda / I_{\gamma} \\
y \in I_{\gamma}}} J_{3}(x-y)=\frac{1}{4} \sum_{i=1}^{v}|\gamma|_{i} J_{3}^{i} .
\end{aligned}
$$

$$
\begin{aligned}
\left\|e^{\frac{\beta K_{A}}{2}} P_{\gamma} R_{\gamma} e^{-\frac{\beta K_{A}}{2}}\right\|_{\mu=\frac{1}{2} J_{3}}^{2} & =e^{2 \beta C_{\gamma}}\left\|e^{\frac{\beta K_{A}}{2}} P_{\gamma} e^{-\frac{\beta K_{A}}{2}} e^{\frac{\beta V_{\gamma}}{2}}\right\|_{\mu=\frac{1}{2} J_{3}}^{2} \\
& =e^{2 \beta C_{\gamma}} .
\end{aligned}
$$


Thus with $|\gamma|_{i}=2 L_{i}$

$$
\varrho_{0}(\beta) \leqq \prod_{i=1}^{v}\left[\sum_{L=1}^{\infty} L^{\frac{1}{v-1}} 3^{2 L-1} e^{\beta L J_{3}^{i}}\right]
$$

and if $v \geqq 2, J_{3}^{i}<0$, then $\varrho_{0}(\beta)$ is strictly smaller than $\frac{1}{2}$ for sufficiently large $\beta$, i.e. there exists a phase transition at very low temperature $(1 / \beta)$ due to the above proposition.

\section{The Anisotropic Heisenberg Model}

The first step in the estimate required for the Heisenberg model is again very easy. One calculates that

$$
\left.R_{\gamma} K_{\Lambda} R_{\gamma}\right|_{\mu=\frac{1}{2} J_{3}}=\left.K_{\Lambda}\right|_{\mu=\frac{1}{2} J_{3}}-T_{\gamma}-V_{\gamma}-C
$$

where the definitions of $V_{\gamma}$ and $\mathrm{C}_{\gamma}$ are the same as in the Ising case and $T_{\gamma}$ is given by

Hence

$$
T_{\gamma}=-\frac{1}{2} \sum_{\substack{x \in A / I_{\gamma} \\ y \in I_{\gamma}}} J_{12}(x-y)\left(a_{x}^{+}-a_{x}\right)\left(a_{y}^{+}-a_{y}\right) .
$$

$$
\left\|e^{\frac{\beta K_{A}}{2}} P_{\gamma} R_{\gamma} e^{-\frac{\beta K_{A}}{2}}\right\|_{\mu=\frac{1}{2} J_{3}}^{2}=e^{2 \beta C_{\nu}}\left\|e^{\frac{\beta K_{\Lambda}}{2}} P_{\gamma} e^{-\frac{\beta}{2}\left(K_{\Lambda}-T_{\gamma}-V_{\gamma}\right)}\right\|_{\mu=\frac{1}{2} J_{3}}^{2} .
$$

Thus we immediately obtain the factor $\exp \left\{2 \beta C_{\gamma}\right\}$ which appears in the Ising calculation and which is essential in establishing that $\varrho_{0}(\beta)$ is small for large $\beta$. However we are still left with a complicated operator whose norm must be estimated. In the Ising case this operator has norm 1 and we will estimate in the present case by using perturbation theory about this point.

Let $A, B, A^{\prime}, B^{\prime}$ and $C$ be matrices and consider the expression

$$
N=e^{A+B} C e^{-A-B+A^{\prime}+B^{\prime}} .
$$

Introduce the function $f$ by the definition

$$
f(t)=e^{(1-t) A} e^{t(A+B)} C e^{-t\left(A+B-A^{\prime}-B^{\prime}\right)} e^{-(1-t)\left(A-A^{\prime}\right)}
$$

and note that $N=f(1)$. We have

$$
\frac{d f(t)}{d t}=e^{(1-t) A} B e^{-(1-t) A} f(t)-f(t) e^{(1-t)\left(A-A^{\prime}\right)}\left(B-B^{\prime}\right) e^{-(1-t)\left(A-A^{\prime}\right)}
$$


and thus defining

we find

$$
B(t)=e^{t A} B e^{-t A}, \quad \hat{B}(t)=e^{t\left(A-A^{\prime}\right)} B e^{-t\left(A-A^{\prime}\right)},
$$

where

$$
f(t)=f(0)+\int_{0}^{t} d t_{1}\left\{\left[B\left(1-t_{1}\right), f\left(t_{1}\right)\right]+f\left(t_{1}\right) X\left(1-t_{1}\right)\right\}
$$

$$
X(t)=B(t)-\hat{B}(t)+\hat{B}^{\prime}(t) .
$$

This integral equation can be iterated to give a formal series solution for $f(t)$ in terms of $f(0)$. Thus we obtain

$$
N=f(0)+\int_{0}^{1} d t\{[B(t), f(0)]+f(0) X(t)\}+\cdots
$$

where we do not explicitly display the higher iterates. The form of these iterates is however clear; at each order terms occur which are either multiple commutators of operators $B\left(t_{1}\right), B\left(t_{2}\right), \ldots$ with $f(0)$, or the multiple product of $f(0)$ with operators $X\left(t_{1}\right), X\left(t_{2}\right)$ etc., or mixtures of commutators and products.

Let us now consider the above formulae with the identification

$$
\begin{aligned}
A & =\frac{\beta}{2}\left(V_{A}-\mu N_{A}\right), & B & =\frac{\beta}{2} T_{A}, \\
A^{\prime} & =\frac{\beta}{2} V_{\gamma}, & B^{\prime} & =\frac{\beta}{2} T_{\gamma}, \\
C & =P_{\gamma}, & \mu & =\frac{1}{2} J_{3} .
\end{aligned}
$$

We find immediately that

$$
f(0)=e^{A} C e^{-A+A^{\prime}}=P_{\gamma} .
$$

Thus $\|f(0)\|=1$. We now proceed to estimate the norms of the higher terms in the series expansion for $N$ and investigate under which conditions this series is convergent. Most of the qualitative features of these estimates occur already with the first order term which we next examine in detail.

We have

$$
\begin{aligned}
& {[B(t), f(0)]} \\
& \quad=\frac{\beta}{4} \sum_{y, x \in \Lambda} J_{12}(x-y)\left[a_{x}^{+} a_{y}, P_{y}\right] \exp \left\{\frac{\beta t}{2} \sum_{z}\left(J_{3}(x-z)-J_{3}(y-z)\right) a_{z}^{+} a_{z}\right\} .
\end{aligned}
$$


Now the commutator occuring on the right hand side vanishes unless $x \in S_{\gamma} \cup S_{\gamma}^{\prime}$ or $y \in S_{\gamma} \cup S_{\gamma}^{\prime}$ and this fact together with the definition of $P_{\gamma}$ and the assumption $J_{3}^{i}<0$ lead directly to the estimate

$$
\|[B(t), f(0)]\| \leqq \beta\left\|J_{12}\right\| e^{\frac{\beta t}{2}\left\|J_{3}\right\|} N_{\gamma} .
$$

Note that this bound is independent of the size of $\Lambda$. Next consider $X(t)$. The definition of $X(t)$ involves the difference of two operators $T_{\Lambda}(t)$ and $\hat{T}_{\Lambda}(t)$ both of which are dependent on the size of $\Lambda$. Clearly the difference however depends only on the size of $\gamma$, a property shared by the third term $\hat{T}_{\gamma}(t)$ which enters in the definition of $X(t)$. A straightforward but laborious calculation leads to the estimate

$$
\|X(t)\| \leqq \frac{7}{2} \beta\left\|J_{12}\right\| e^{\frac{3 \beta t}{2}\left\|J_{3}\right\|} N_{\gamma} .
$$

This estimate is extremely crude but our principal interest is its qualitative form.

Combining the above calculations we have

$$
\left\|\int_{0}^{1} d t\{[B(t), f(0)+f(0) X(t)]\}\right\| \leqq \frac{8}{3} \frac{\left\|J_{12}\right\|}{\left\|J_{3}\right\|}\left(e^{\frac{3 \beta}{2}\left\|J_{3}\right\|}-1\right) N_{\gamma} .
$$

Now consider the higher terms occuring in the series expansion under consideration. Repetition of the above arguments gives

$$
\left\|\left[B\left(t_{1}\right)\left[\ldots\left[B\left(t_{n}\right), f(0)\right]\right]\right]\right\| \leqq\left(2 \beta\left\|J_{12}\right\|\right)^{n} e^{\frac{\beta}{2}\left(t_{1}+\cdots+t_{n}\right)\left\|J_{3}\right\|} \prod_{p=0}^{n-1}\left(N_{\gamma}+4 v p\right),
$$

i.e. each additional commutator introduces a factor

$$
\beta\left\|J_{12}\right\| \exp \left\{\beta t\left\|J_{3}\right\| / 2\right\}
$$

and the number of terms which are not indentically zero due to the commutation relations is majorized by $2^{n}$ times the $N_{\gamma}$ dependent product. Also

$$
\left\|f(0) X\left(t_{1}\right) \ldots X\left(t_{n}\right)\right\| \leqq\left(\frac{7}{2} \beta\left\|J_{12}\right\| N_{\gamma}\right)^{n} e^{\frac{3 \beta}{2}\left(t_{1}+\cdots+t_{n}\right)}
$$

and it remains to consider the mixed terms occuring in the iteration. The qualitative behaviour of these terms is very similar to the terms discussed; the dependence on the size of $\Lambda$ is suppressed by the commutators; there occur $n$ factors proportional to $2 \beta\left\|\mathrm{J}_{12}\right\|$ and the worst proportionality factor is $7 / 2$; there occur factors of the form $\exp \left\{a \beta\left\|J_{3}\right\| t_{i}\right\}$ and the largest possible value of a is $3 / 2$; finally there 
occurs an $N_{\gamma}$ dependent factor which is certainly majorized by $\left(2 N_{\gamma}\right.$ $+4 n v)^{n}$. At the $n$-th order there are $2^{n}$ terms in the iteration formula and hence we find after integration that the norm of the $n$-th order term is majorized by

$$
\left(\frac{28}{3} \frac{\left\|J_{12}\right\|}{\left\|J_{3}\right\|}\left(e^{\frac{3 \beta}{2}\left\|J_{3}\right\|}-1\right)\right)^{n} \frac{\left(2 N_{\gamma}+4 v n\right)^{n}}{n !} .
$$

There are two important features of this estimate. Firstly it follows that for fixed $\beta$ the iteration expansion is convergent if the anisotropy parameter $\left\|J_{12}\right\| /\left\|J_{3}\right\|$ is sufficiently small. Secondly the radius of convergence is independent of $N_{\gamma}$. These features alone are important in establishing the existence of a phase transition and not the numerical coefficients which we have crudely overestimated. Thus using the inequality $x^{n} \leqq n ! e^{x}$ we deduce that there exist positive numbers $a$ and $b$ such that

$$
\left\|e^{\frac{\beta K_{A}}{2}} P_{\gamma} e^{\frac{\beta}{2}\left(K_{A}-T_{\gamma}-V_{\nu}\right)}\right\|_{\mu=\frac{1}{2} J_{3}} \leqq e^{\frac{a}{2} N_{\gamma}}\left[1-b \frac{\left\|J_{12}\right\|}{\left\|J_{3}\right\|}\left(e^{\frac{3 \beta}{2}\left\|J_{3}\right\|}-1\right)\right]^{-1}
$$

for all values of $\left\|J_{12}\right\|,\left\|J_{3}\right\|$, and $\beta$, such that the right hand side is positive. Hence we have a bound of the term

$\varrho_{0}(\beta) \leqq\left[\prod_{i=1}^{v}\left\{\sum_{L=1}^{\infty} L^{\frac{1}{\nu-1}} 3^{2 L-1} e^{L\left(\beta J_{3}^{i}+a\right)}\right\}\right]\left[1-b \frac{\left\|J_{12}\right\|}{\left\|J_{3}\right\|}\left(e^{\frac{3 \beta}{2}\left\|J_{3}\right\|}-1\right)\right]^{-2}$.

If $v \geqq 2$ and $J_{3}^{i}<0$, we may conclude that there exists a set of values of $\beta$ and $\left\|J_{12}\right\| /\left\|J_{3}\right\|$ such that $\varrho_{0}(\beta)$ is strictly smaller than $\frac{1}{2}$, i.e. there exists a phase transition due to the proposition of Section 2. We summarise the information as follows.

Theorem. There exists an open subset $P \subset R^{2}$ with non-zero Lebesgue measure such that if $J_{3}^{i}<0(i=1, \ldots, v ; v \geqq 2)$ and $\left(\beta,\left\|J_{12}\right\| /\left\|J_{3}\right\|\right) \in P$ then the anisotropic Heisenberg model exhibits two phases; $P$ is convex in $\beta$, and convex in $\frac{\left\|J_{12}\right\|}{\left\|J_{3}\right\|}$ for fixed $J_{3}^{i}$.

Quantitative estimates of the size of $P$ can be obtained by the above methods. Note that our result has one seemingly unphysical feature. For small non-zero values of $\left\|J_{12}\right\| /\left\|J_{3}\right\|$ we can establish that a phase transition takes place for some large value $\beta_{c}$ of $\beta$, i.e. the spontaneous magnetization is non-zero. However our estimates do not allow us to conclude that there is a non-zero magnetization for all $\beta>\beta_{c}$. It would be interesting to study whether $\beta_{c}$ is necessarily a single-valued increasing function of the anisotropy parameter. 


\section{Concluding Remarks}

In the above we have actually considered a partially anisotropic spin coupling. However our methods straightforwardly extend to the fully anisotropic Hamiltonian

$$
\begin{aligned}
& H_{\Lambda}=\frac{1}{8} \sum_{x, y \in \Lambda}\left\{J_{1}(x-y) \sigma_{x}^{(1)} \sigma_{y}^{(1)}+J_{2}(x-y) \sigma_{x}^{(2)} \sigma_{y}^{(2)}+J_{3}(x-y) \sigma_{x}^{(3)} \sigma_{y}^{(3)}\right\} \\
& +h \sum_{x \in \Lambda} \sigma_{x}^{(3)}
\end{aligned}
$$

and similar results can be stated; with nearest neighbour couplings of $J_{1}, J_{2}$, and $J_{3}$, phase transitions take place if $\left\|J_{1}\right\| /\left\|J_{3}\right\|$ and $\left\|J_{2}\right\| /\left\|J_{3}\right\|$ are both small.

Recently Dobrushin [5] has extended the Peierls argument to establish that there exists a region, in the $(\beta, \mu)$ plane, of two phases for the repulsive Ising model $\left(J_{3}^{i}>0\right)$. Dobrushin's arguments can be generalized in a manner similar to the preceding to conclude that the same property is shared by the strongly anisotropic Heisenberg model. In the repulsive case an estimation of the magnetization of a sub-lattice has to be made and this is again done by establishing an upper bound in terms of a series summed over cycles. However one then uses an estimate of the form

$$
\sum_{X ; \Gamma(X) \ni \gamma}\left\langle X\left|e^{-\beta K_{A}}\right| X\right\rangle \leqq\left\|e^{\frac{\beta K_{A}}{2}} U_{\Lambda} P_{\gamma} R_{\gamma} e^{-\frac{\beta K_{A}}{2}}\right\|^{2} \operatorname{Tr}_{\mathscr{H}_{\Lambda}}\left(e^{-\beta K_{A}}\right)
$$

This is valid for any unitary operator $U_{A}$ on $\mathscr{H}_{A}$ and a suitable choice has to be made.

Acknowledgements. The major part of the above work was carried out in July 1968 whilst the author was a guest of the Seminar für Theoretische Physik, ETH, Zürich; the hospitality of Prof. R. Jost during this period is warmly acknowledged.

\section{References}

1. Peierls, R.: Proc. Cambridge Phil. Soc. 32, 477 (1936).

2. Dobrushin, R. L.: Teorija Verojatnostei ill Primeneniya 9, 626 (1964).

3. Griffiths, R.: Phys. Rev. 136, A 437 (1964).

4. Ginibre, J., A. Grossman, and D. Ruelle: Commun. Math. Phys. 3, 187 (1966).

5. Dobrushin, R. L.: Functional Analysis 2, 44 (1968).

D. W. Robinson Physique Théorique, C.N.R.S.

31, Chemin J. Aiguier

13 Marseille 9è, France 